\title{
Malignant transformation of liver fatty acid binding protein- deficient hepatocellular adenomas: histopathologic spectrum of a rare phenomenon
}

\author{
Juan Putra $\mathbb{1}^{1,2} \cdot$ Linda D. Ferrell ${ }^{3} \cdot$ Annette S. H. Gouw $^{4} \cdot$ Valerie Paradis $^{5} \cdot$ Arvind Rishi $^{6} \cdot$ Christine Sempoux $^{7}$. \\ Charles Balabaud $^{8} \cdot$ Swan N. Thung ${ }^{1} \cdot$ Paulette Bioulac-Sage ${ }^{8,9}$
}

Received: 15 July 2019 / Revised: 10 September 2019 / Accepted: 10 September 2019 / Published online: 30 September 2019

(c) The Author(s), under exclusive licence to United States \& Canadian Academy of Pathology 2019

\begin{abstract}
The molecular classification of hepatocellular adenomas highlights a distinctive genotype-phenotype correlation. Malignant transformation is an exceptionally rare complication of hepatocyte nuclear factor $1 \alpha$ (HNF1A)-inactivated hepatocellular adenomas. This subtype is characterized by loss of liver fatty acid binding protein immunoexpression. In this study, we characterized the histopathologic spectrum of 13 liver fatty acid binding protein-deficient hepatocellular adenoma cases showing malignant transformation from multiple centers. Clinicopathologic characteristics of these patients were evaluated. Stains for reticulin, liver fatty acid binding protein, beta-catenin and glutamine synthetase were applied to these lesions. Moreover, the findings were compared to patients with $\beta$-catenin mutated hepatocellular adenoma. Liver fatty acid binding protein-deficient hepatocellular adenomas with borderline features/carcinoma were seen predominantly in females $(77 \%)$ with an average age of $46 \pm 18$ years and multiple lesions (77\%; five patients with adenomatosis). Meanwhile, $\beta$-catenin mutated hepatocellular adenoma patients with malignant transformation were predominantly male $(67 \%, p=0.018)$ with single lesion $(86 \%, p=0.0009)$. The largest liver fatty acid binding protein-deficient hepatocellular adenoma nodule in each patient ranged from 4 to $15.5 \mathrm{~cm}$. Loss of liver fatty acid binding protein by immunohistochemistry was noted in all adenoma and borderline/carcinoma components. Features of malignant transformation were pseudoglandular architecture $(85 \%)$, cytologic atypia (85\%), architectural atypia (100\%) and lack of steatosis $(100 \%)$. Other findings included myxoid change (39\%), peliosis (46\%) and sinusoidal dilatation (46\%). Molecular studies confirmed somatic inactivation of HNF1A in 3 cases and absence of TERT promotor and exon 3 CTNNB1 mutations in five cases. To summarize, liver fatty acid binding protein-deficient hepatocellular adenoma with malignant transformation is most frequently seen in female patients with multiple lesions. Most of these lesions demonstrate pseudoglandular architecture, cytologic and architectural atypia, with lack of steatosis. The natural history of these lesions is relatively benign with the exception of disease recurrence in 1 patient.
\end{abstract}

Supplementary information The online version of this article (https:// doi.org/10.1038/s41379-019-0374-x) contains supplementary material, which is available to authorized users.

Juan Putra

Juan.Putra@sickkids.ca

1 Department of Pathology, Icahn School of Medicine at Mount Sinai, New York, NY, USA

2 Division of Pathology, Department of Paediatric Laboratory Medicine, Hospital for Sick Children, Toronto, ON, Canada

3 Department of Pathology, University of California, San Francisco, San Francisco, CA, USA

4 Department of Pathology, University of Groningen, University Medical Center Groningen, Groningen, The Netherlands
5 Pathology Department, Beaujon Hospital, Clichy, France

6 Department of Pathology, Long Island Jewish Medical Center, New Hyde Park, NY, USA

7 Service of Clinical Pathology, Lausanne University Hospital, Institute of Pathology, Lausanne, Switzerland

8 BaRITOn Bordeaux Research in Translational Oncology, Univ Bordeaux, INSERM UMR1053, F-33000 Bordeaux, France

9 Department of Pathology, CHU Bordeaux, F-33000 Bordeaux, France 


\section{Introduction}

Hepatocellular adenomas are uncommon benign hepatic lesions classically seen in young women taking oral contraceptives. The molecular and pathological classification of hepatocellular adenomas has demonstrated a distinctive genotype-phenotype correlation [1]. This classification is clinically significant as each subtype is associated with certain risk factors and complications, such as bleeding and malignant transformation [2]. Malignant transformation of hepatocellular adenomas is most frequently identified in males, patients taking anabolic steroids, lesions greater than $5 \mathrm{~cm}$ and hepatocellular adenomas with $\beta$-catenin mutation secondary to activating mutations involving the exon 3 of cadherin associated protein $\beta 1$ $(C T N N B 1)$ gene $[2,3]$.

Hepatocyte nuclear factor $1 \alpha(H N F 1 A)$-inactivated hepatocellular adenoma is molecularly defined by inactivating mutations of $H N F 1 A$ (or $T C F 1$ ) gene [2, 4]. This subtype comprises $35-40 \%$ of all hepatocellular adenomas and the mutations are predominantly somatic in origin [4]. Meanwhile, the less frequent germline mutations are associated with hepatic adenomatosis (greater than 10 lesions in the liver) and maturity-onset diabetes of the young type 3 [5]. Although HNF1A-inactivated hepatocellular adenoma is not usually associated with malignant transformation, rare cases have been previously reported [6-9]. Liver fatty acid binding protein is a downstream target of HNF1A, and loss of liver fatty acid binding protein by immunohistochemistry is routinely used to identify HNF1A-inactivated hepatocellular adenomas $[2,4]$. In this series, we use the term liver fatty acid binding protein-deficient hepatocellular adenoma due to the lack of molecular confirmation of HNF1A inactivation in most cases. We aimed to evaluate the histopathologic spectrum of liver fatty acid binding protein-deficient hepatocellular adenomas showing features of malignant transformation.

\section{Materials and methods}

Cases of liver fatty acid binding protein-deficient hepatocellular adenoma with histologic features of malignant transformation, including those with features of borderline lesions and hepatocellular carcinoma, were identified from multiple institutions in the United States and Europe. These cases were selected on the basis of loss of liver fatty acid binding protein immunoexpression. Limited demographic information (sex and age) and clinical history (past medical history, family history, presenting symptoms and the type of management) was obtained, as most cases were consultation cases sent to the pathology department. The number and size of hepatocellular adenomas provided by participating institutions were based on the imaging studies and/or pathologic examinations.

The original diagnosis was rendered by the contributing pathologist from each institution. Histologic slides for these cases were evaluated by each contributing pathologist. Key representative section(s) for each case was reviewed by two liver pathologists (J.P. and S.N.T.). Routine hematoxylin and eosin (H\&E)-stained slides of formalin fixed tissues were assessed for the following features: the predominant type of steatosis (macrovesicular or microvesicular), features of malignant transformation, myxoid change, peliosis, and sinusoidal dilatation. These histologic findings were considered focal when they occupied $<50 \%$ of each lesion and diffuse if $50 \%$ or greater involvement was identified.

Histologic characteristics of malignant transformation included pseudoglandular architecture/rosette formation, cytologic, and architectural atypia. Cytologic atypia was characterized by small cell change in which hepatocytes demonstrating increased nuclear to cytoplasmic ratio, mild nuclear pleomorphism, hyperchromatic nuclei, and cytoplasmic basophilia [10]. Findings of architectural atypia included thickened hepatocyte plates (three hepatocytes or more) and/or nodule-within-nodule appearance. In cases demonstrating overt malignant features, hepatocellular carcinoma differentiation was graded using Edmondson and Steiner system based on the nuclear features [11].

Special stain and immunohistochemical studies, performed at original institutions, included reticulin stain, liver fatty acid binding protein, beta-catenin, and glutamine synthetase. A subset of cases was also evaluated with CD34 and glypican-3 immunostains. The immunohistochemical panel for hepatocellular adenoma evaluation at the primary authors' institution (New York) included liver fatty acid binding protein (2G4, Abcam, 1:1000 dilution), beta catenin (Agilent, ready-to-use), glutamine synthetase (BD transduction laboratory, 1:100 dilution), and CD34 (EP373Y, Abcam, 1:100 dilution) immunostains. Molecular testing results were shown when available.

Patients with malignant transformation of $\beta$-catenin mutated hepatocellular adenoma diagnosed at the primary authors' institutions (New York) from 2000-17 were selected as control group. In addition, review of the literature (2006-18) was performed to identify additional cases of $\beta$-catenin mutated hepatocellular adenoma with malignant transformation. Only case series and reports with detailed demographic and pathologic information were included in the control group. The demographic and pathologic information was collected for comparison. Categorical and continuous data was analyzed using Fisher's exact test and $t$-test, respectively. 
Table 1 Clinical information of patients with liver fatty acid binding protein-deficient hepatocellular adenoma showing features of malignant transformation

\begin{tabular}{llllll}
\hline Patient & Sex & Age (years) & Past or family medical history & Presenting symptoms & Type of surgery \\
\hline 1 & F & 57 & & Abnormal LFT & Partial resection \\
2 & F & 52 & Possible OWR syndrome & Abnormal LFT & Partial resection \\
3 & M & 34 & - & Abdominal pain & Partial resection \\
4 & M & 27 & Tetralogy of Fallot & Abnormal LFT & OLT \\
5 & F & 53 & - & Unknown & Partial resection \\
$6[27]$ & F & 55 & Budd-Chiari syndrome & Unknown & OLT \\
$7[27]$ & M & 23 & Budd-Chiari syndrome & Unknown & OLT \\
$8[6]$ & F & 23 & MODY type 3 & Abdominal pain & Partial resection \\
$9[8]$ & F & 60 & Type 2 diabetes mellitus; Deceased daughter with HCA & Abdominal pain & Partial resection \\
10 & F & 55 & - & Elevated LFT & Partial resection \\
11 & F & 18 & - & Abdominal pain & Partial resection \\
12 & F & 75 & - & Slightly elevated AFP & Partial resection \\
13 & F & 64 & - & Unknown & Partial resection \\
\hline
\end{tabular}

$F$ female, $M$ male, $O W R$ Osler-Weber-Rendu, $M O D Y$ maturity onset of diabetes of the young, $H C A$ hepatocellular adenoma, $L F T$ liver function tests, $A F P$ alpha fetoprotein, $O L T$ orthotopic liver transplantation

\section{Results}

\section{Patient demographics}

After review of the key sections (H\&E, special, and immunohistochemical stains), 13 cases of liver fatty acid binding protein-deficient hepatocellular adenoma with features of malignant transformation were included in the study. The patient cohort was comprised of ten females (77\%) and three males (23\%) ranging from 18 to 75 years old (average age of $46 \pm 18$ years). Pertinent past medical history included diabetes (one patient with maturity-onset diabetes of the young type 3 and one patient with type 2 diabetes mellitus) and Budd-Chiari syndrome (two patients). In addition, one of the patients had a deceased daughter who was diagnosed with hepatocellular adenoma. Of available information, abdominal pain (44\%) and abnormal liver function tests (44\%) were the predominant presenting symptoms. All patients presented with hepatic lesion(s) without any evidence of metastatic disease. The majority of patients underwent partial hepatectomy (77\%), while three patients (23\%) underwent orthotopic liver transplantation (OLT). The clinical information was summarized in Table 1.

\section{Pathologic features}

Ten patients (77\%) had multiple lesions; hepatic adenomatosis (ten lesions or more) was noted in five of these patients $(39 \%)$. The average size of the largest nodule for each patient was $8.9 \pm 3.5 \mathrm{~cm}$ (range: $4-15.5 \mathrm{~cm}$ ). Microscopically, the hepatocellular adenomas were characterized by well-differentiated hepatocellular lesions showing mildly thickened hepatocyte plates and unpaired arterioles. Most hepatocellular adenomas (Figs. 1a and 2a) demonstrated focal predominantly macrovesicular steatosis $(85 \%)$, while two lesions (15\%) showed a near complete lack of steatosis. Table 2 summarized the histopathologic characteristics of these cases.

Features of malignant transformation (Figs. 1b-h and 2b) such as pseudoglandular architecture $(85 \%)$ and cytologic atypia (85\%) were noted in most lesions, while architectural atypia was consistently noted in all cases $(100 \%)$. These features were identified in the largest nodule of patients with multiple hepatocellular adenomas. In cases with overt features of malignant transformation, the neoplastic cells showed nuclear features consistent with well-differentiated hepatocellular carcinoma (100\%). In addition, foci of borderline lesion or hepatocellular carcinoma consistently demonstrated lack of or minimal steatosis (100\%). Myxoid change was noted in five cases (39\%), mostly showing focal distribution. Focal peliosis and sinusoidal dilatation were identified in six lesions (46\%).

Reticulin stain was performed in eleven cases; reticulin staining was decreased in the malignant transformation areas of all cases (100\%) (Fig. 2c, d) and was intact in the background hepatocellular adenomas. The diagnosis of liver fatty acid binding protein-deficient hepatocellular adenoma was established on the basis of negative liver fatty acid binding protein immunoexpression in the adenoma component. Loss of liver fatty acid binding protein expression by immunohistochemistry was noted in both adenoma and borderline/carcinoma components of all cases (100\%) (Fig. 2e, f). CD34 immunostain was 


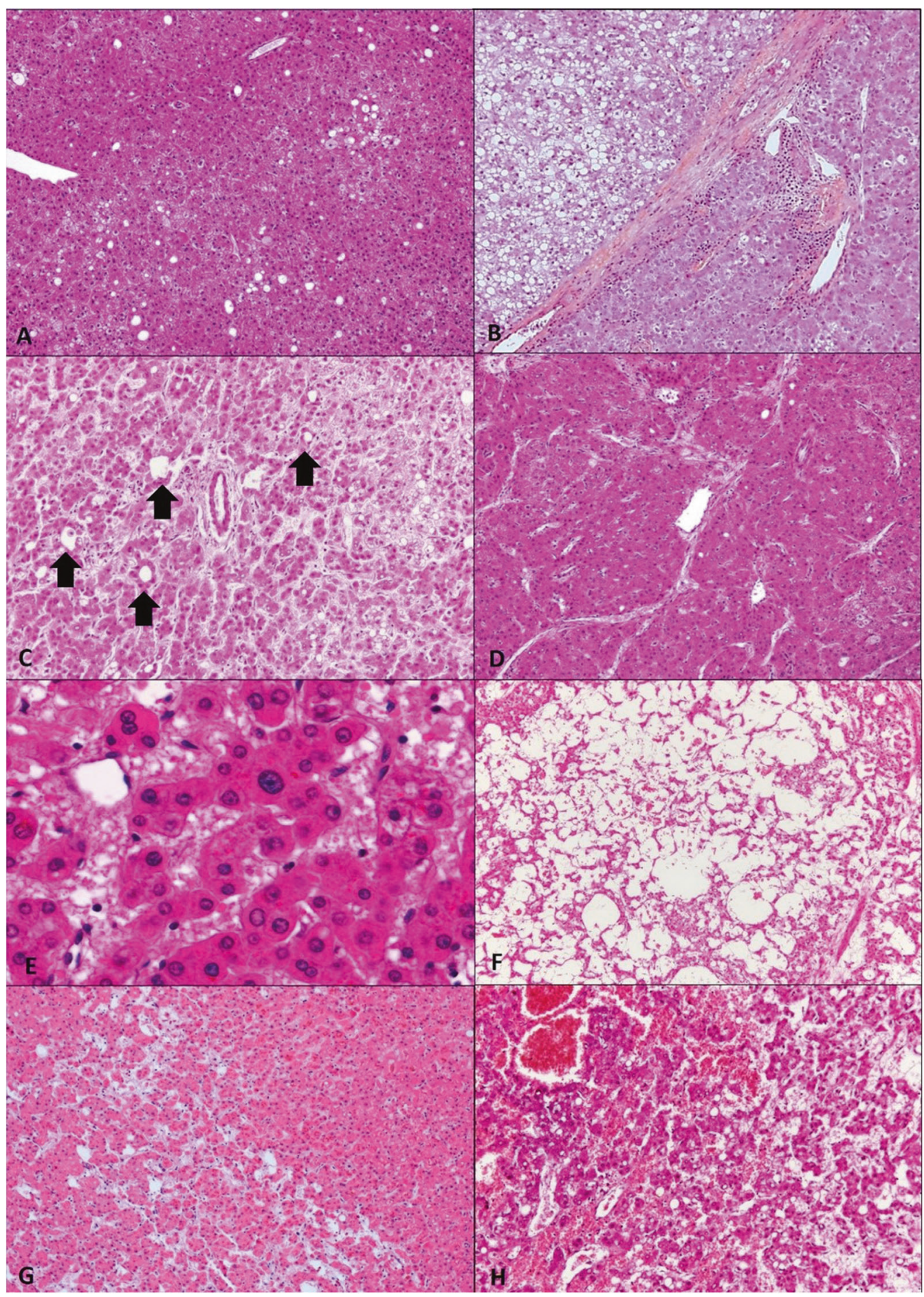

Fig. 1 a Liver fatty acid binding protein-deficient hepatocellular adenoma is characterized by a well-differentiated hepatocellular neoplasm with scattered macrovesicular steatosis and unpaired arterioles (patient 3, H\&E, 10x), b low-power-view of interface area between hepatocellular adenoma component on the left (abundant steatosis) and hepatocellular carcinoma on the right (less steatosis with thickened hepatocyte plates) (patient $1, \mathrm{H} \& \mathrm{E}, 4 \times$ ). Features of malignant transformation include $\mathbf{c}$ prominent pseudoglandular architecture (arrows) and small cell change (patient 7, H\&E, 10x) with d architectural atypia in a form of thickened trabeculae (patient 6, H\&E, 10×); e nuclear pleomorphism is also identified in the hepatocellular carcinoma component (patient $6, \mathrm{H} \& \mathrm{E}, 40 \times$ ). Other histologic findings noted are $\mathbf{f}$ myxoid change (patient 5, H\&E, 10×), g sinusoidal dilatation or telangiectasia (patient $9, \mathrm{H} \& \mathrm{E}, 10 \times$ ) and $\mathbf{h}$ peliosis as seen on the upper left side (patient 5, H\&E, 10x) 


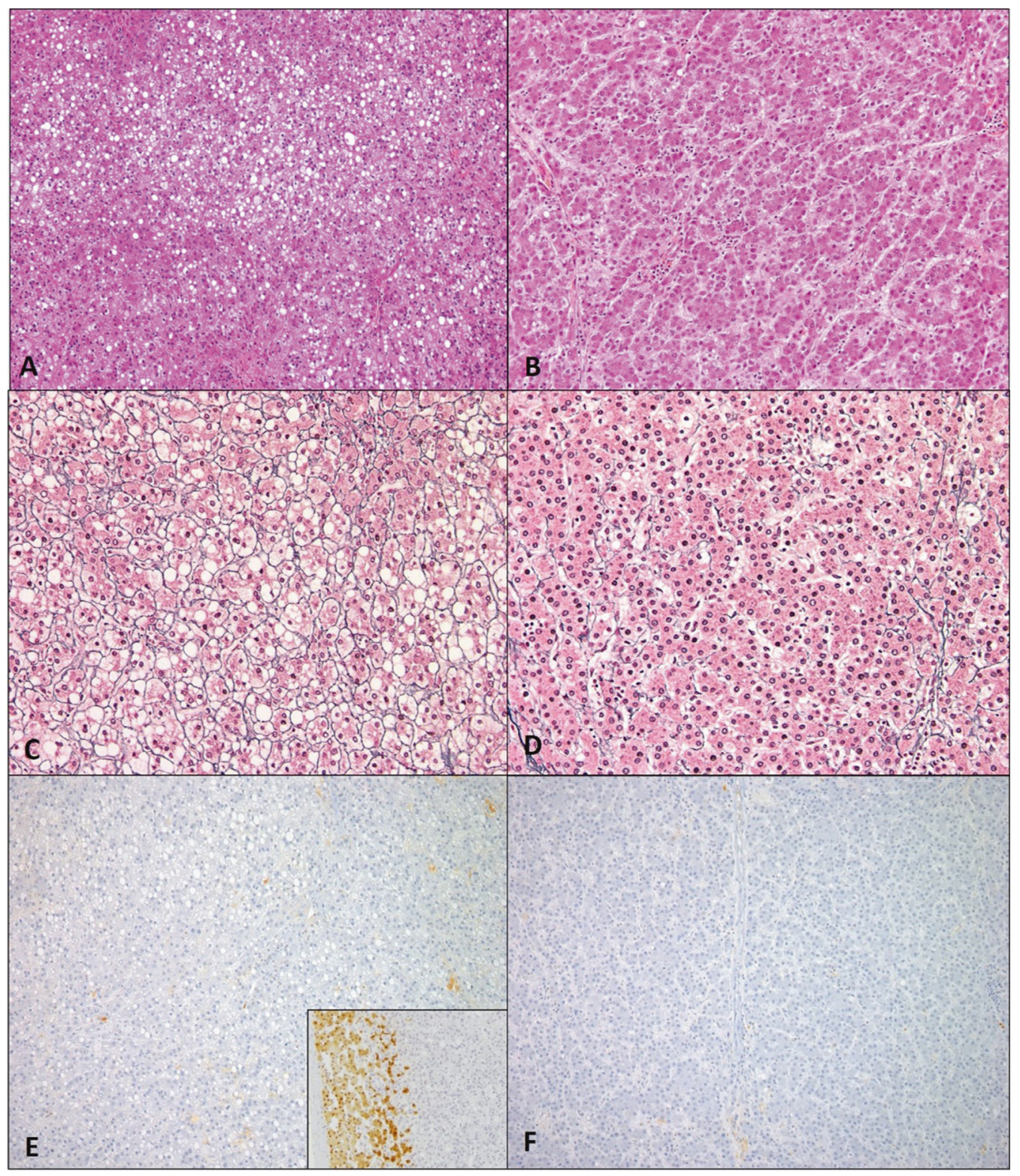

Fig. 2 Hepatic lesion of patient 1 demonstrates areas consistent with a hepatocellular adenoma $(\mathrm{H} \& \mathrm{E}, 10 \times)$ and $\mathbf{b}$ well-differentiated hepatocellular carcinoma; note absence of steatosis in the malignant component $(\mathrm{H} \& \mathrm{E}, 10 \times)$. c Preserved reticulin framework despite macrovesicular steatosis is noted in the hepatocellular adenoma component (reticulin, 10x), meanwhile $\mathbf{d}$ disrupted reticulin framework with associated thickened trabeculae is seen in the area of

hepatocellular carcinoma (reticulin, 10x). Liver fatty acid binding protein is not expressed in the e hepatocellular adenoma (liver fatty acid binding protein, $10 \times$ ) and $\mathbf{f}$ hepatocellular carcinoma component (liver fatty acid binding protein, 10x). Inset e highlights normal expression of liver fatty acid binding protein in the adjacent nonneoplastic liver tissue (liver fatty acid binding protein, 10x)

performed in nine cases, and eight of these cases (89\%) showed diffuse increased staining in the borderline/carcinoma areas (Fig. 3a). Meanwhile, minimally increased CD34 staining pattern was identified in the adenoma components (Fig. 3b). The glutamine synthetase immunostain showed heterogenous distribution ranging from negative (39\%), focal (39\%), and diffuse expression $(23 \%)$ in the areas of malignant transformation; the focal and diffuse glutamine synthetase staining demonstrated heterogeneous (weak to moderate) strength of expression
(Fig. 3c). Nuclear beta-catenin expression was absent in both adenoma and borderline/carcinoma components of all cases (100\%). Glypican-3 immunohistochemical stain was only positive in one of six hepatocellular carcinomas $(17 \%)$.

\section{Molecular findings}

Five cases were submitted for molecular testing. Somatic inactivating HNF1A mutation was confirmed by Sanger 


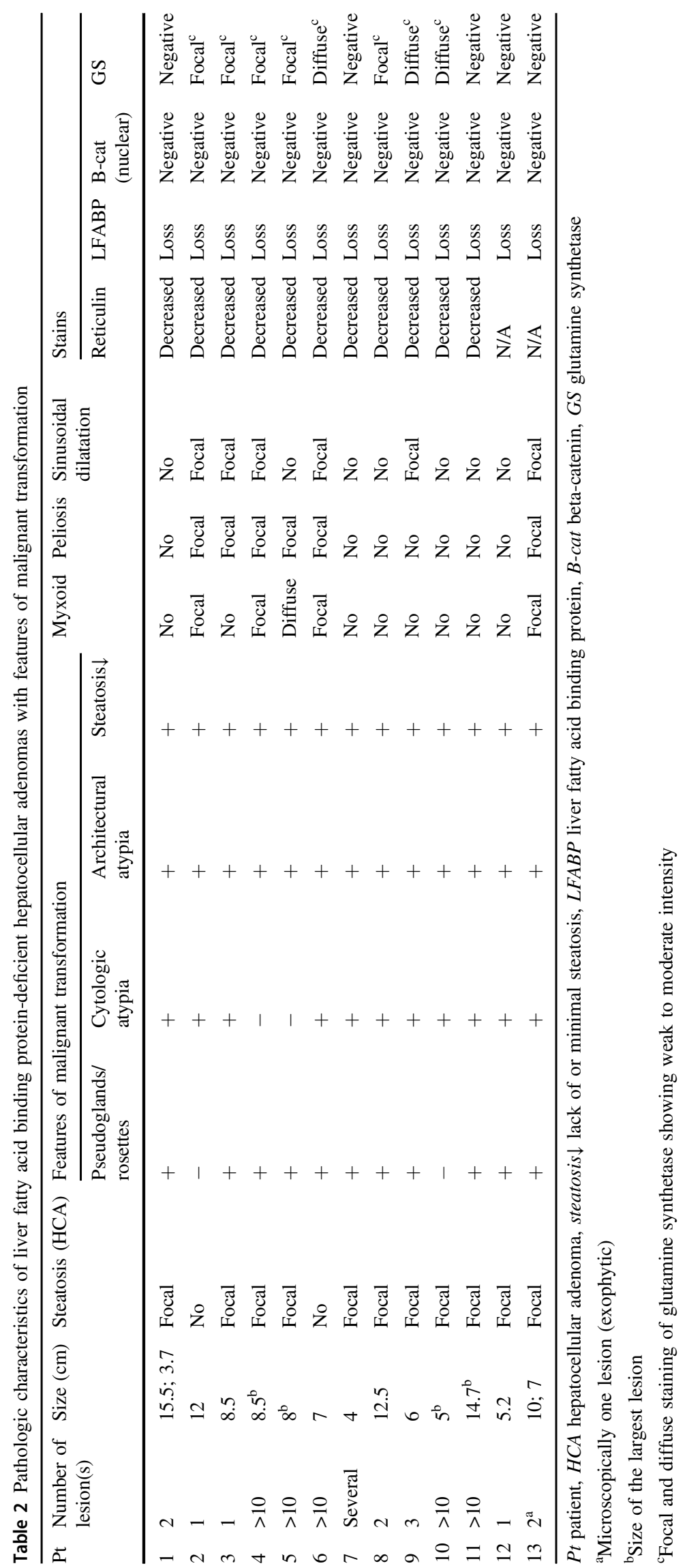




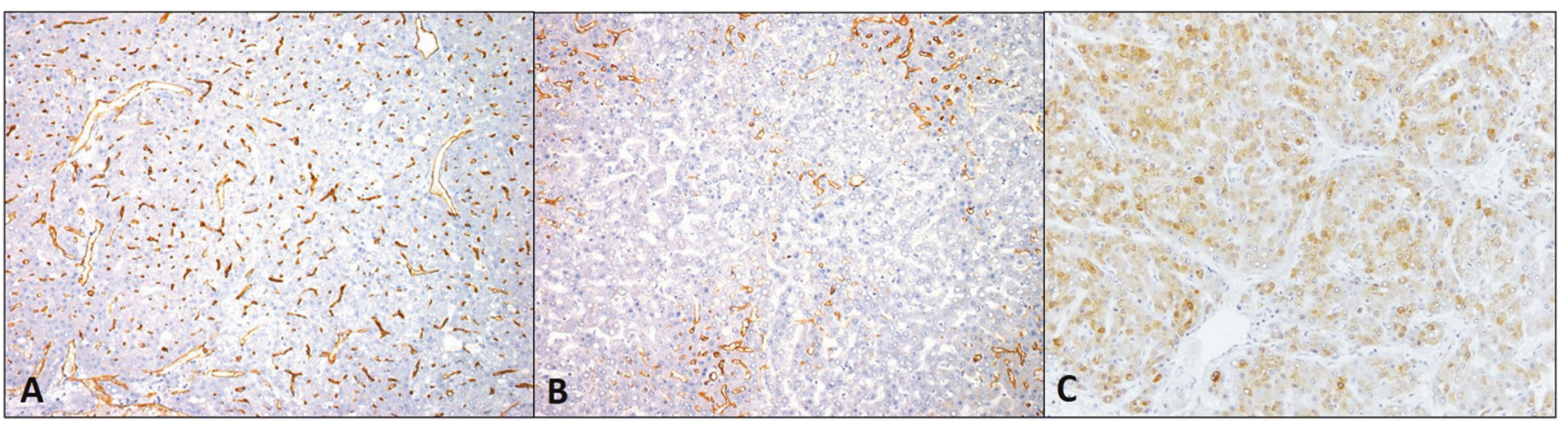

Fig. 3 Diffuse increase of CD34 immunoexpression is noted in a the hepatocellular carcinoma component (patient 6, CD34, 10x), while mildly increased staining pattern is observed in $\mathbf{b}$ the hepatocellular adenoma component (patient 6, CD34, 10x). c Diffuse, moderate and heterogeneous glutamine synthetase staining is noted in the hepatocellular carcinoma component of patient 6 (glutamine synthetase, $10 \times$ )

Table 3 Comparison between patients with liver fatty acid binding protein-deficient hepatocellular adenoma and $\beta$-catenin mutated hepatocellular adenoma showing malignant transformation

\begin{tabular}{llll}
\hline Characteristics & LFABP-deficient hepatocellular adenomas & $\beta$-catenin mutated hepatocellular adenomas ${ }^{\mathrm{a}}[12-22]$ & $P$ value \\
\hline Number of patients & 13 & $10(33 \%)$ & $44(20-66)$ \\
Number of female & $10(77 \%)$ & Androgen/steroid, OCP, \\
Mean age (range; years) & $46(18-75)$ & GSD type 1 \\
Risk factors & BCS, MODY type 3, DM & $3(16 \%)$ \\
& $10(77 \%)$ & $2-34$ \\
Multiple adenomas & $4-15.5$ & Well-differentiated (67\%), moderately- \\
Lesion size (range; cm) & Well-differentiated (100\%) & differentiated (27\%), poorly differentiated $(6 \%)$ \\
HCC grade & & 4 cases from primary authors institution; \\
Others & 5 cases with adenomatosis & 26 cases from the literature \\
\hline
\end{tabular}

$H C C$ hepatocellular carcinoma, $L F A B P$ liver fatty acid binding protein, BCS Budd-Chiari syndrome, $M O D Y$ maturity-onset diabetes of the young, $D M$ diabetes mellitus, $O C P$ oral contraceptive pills, GSD glycogen storage disease

${ }^{a} \beta$-catenin mutated hepatocellular adenoma cases included 4 cases from primary authors' institution and 26 cases from the literature [12-22] (see Supplementary Table 1 for details)

sequencing on cases 2, 3, and 4 with no evidence of molecular features of hepatocellular adenoma of inflammatory subtype, TERT promotor and CTNNBI mutations. In case 8, TERT promoter and exon 3 of CTNNB1 gene mutations were not detected by Sanger sequencing. Meanwhile, targeted next generation sequencing of case $\mathbf{1 3}$ showed mutation of GNAS exon 8: c.602G>A; p.R201H. with no evidence of TERT promoter or CTNNB1 mutations. GNAS mutation was noted in both hepatocellular adenoma and hepatocellular carcinoma components. HNF1A was not molecularly tested, as the gene was not part of the limited sequencing panel.

\section{Follow-up information}

Follow-up information was available in five patients. Patient 9 developed multiple recurrences 3 years after initial presentation. Patient 2 developed lung adenocarcinoma with brain and bone metastases 16 years after partial hepatic resection. Meanwhile, patients 3, 4, and 12 were alive with no recurrence at the time of last follow-up (5-year follow up interval for patients 3 and 4; 4-year follow up interval for patient 12).

\section{Patients with malignant transformation of $\beta$-catenin mutated hepatocellular adenoma}

Four patients were diagnosed with $\beta$-catenin mutated hepatocellular adenoma showing features of malignant transformation at primary authors' institution (New York); they were predominantly male ( $75 \%$ male and $25 \%$ female) with an average age of $50 \pm 9$ years at the time of diagnosis (range: 34-56 years). Two of the patients (50\%) took androgen/anabolic steroids in the past. Most patients had single lesions (75\%), while one patient had two hepatocellular adenomas. The size of the largest lesion of each patient ranged from 4.5 to $16 \mathrm{~cm}$. Three of these patients (75\%) showed overlap $\beta$-catenin mutated hepatocellular 
adenoma with inflammatory subtype. All hepatocellular carcinomas were well-differentiated. Two of these patients had follow-up information (1 year and 9 years) with no evidence of recurrent disease.

Twenty-six additional $\beta$-catenin mutated hepatocellular adenoma patients with malignant transformation previously reported in the literature were included in the analysis [12$22]$. These patients were mostly male $(65 \%$ male and $35 \%$ female) with an average age of $42 \pm 14$ years at the time of diagnosis (range: 20-66 years). Associated risk factors included contraceptive pills (four patients) and glycogen storage disease type 1 (two patients). Thirteen of 15 cases with available information (87\%) showed solitary lesions. The size of the lesion ranged from 2 to $34 \mathrm{~cm}$. Most hepatocellular carcinomas demonstrated well differentiation (55\%), whereas $36 \%$ and $9 \%$ of the cases showed moderate and poor differentiation, respectively. The complete clinical and pathological data of the control group was summarized in Supplementary Table 1.

Table 3 highlighted the key differences between liver fatty acid binding protein-deficient hepatocellular adenomas $(n=$ $13)$ and $\beta$-catenin mutated hepatocellular adenomas $(n=30)$ with malignant transformation.

\section{Discussion}

The current molecular classification divides hepatocellular adenomas into six major subgroups with specific clinical and pathological features: HNF1A-inactivated hepatocellular adenoma, inflammatory hepatocellular adenoma, $\beta$-catenin mutated hepatocellular adenoma (exon 3 or exon 7/8), overlap of inflammatory and $\beta$-catenin mutated hepatocellular adenoma, hepatocellular adenoma with sonic hedgehog activation identified by immunohistochemical expression of argininosuccinate synthase 1 (ASS1), and a very small group of unclassified hepatocellular adenomas [2, 23]. In clinical practice, molecular subtyping of hepatocellular adenomas is important for prognostication and aiding in treatment decisions. The Clinical Practice Guidelines by the European Association for the Study of the Liver recommends resection treatment for hepatocellular adenomas greater than $5 \mathrm{~cm}$ or increasing in size, $\beta$-catenin mutated hepatocellular adenoma and hepatocellular adenomas in men irrespective of size; whereas HNF1A-inactivated hepatocellular adenomas $(<5 \mathrm{~cm})$, inflammatory hepatocellular adenoma, and hepatocellular adenomas with negative beta-catenin mutations can be managed conservatively [24].

Herein, we reported the largest series of liver fatty acid binding protein-deficient hepatocellular adenoma with features of malignant transformation. We used the term liver fatty acid binding protein-deficient hepatocellular adenoma, as most of our cases did not have molecular confirmation of
HNF1A inactivating mutations. In contrast to $\beta$-catenin mutated hepatocellular adenoma, malignant transformation is not frequently identified in liver fatty acid binding protein-deficient hepatocellular adenoma. Our study highlights the histopathologic spectrum of this rare but important complication of liver fatty acid binding proteindeficient hepatocellular adenomas. The authors recognize the challenging nature of determining the presence of malignant transformation in hepatocellular adenomas. The histologic assessment is often subjective and the diagnostic criteria are not well established. The evaluation is particularly difficult in the so-called borderline lesions. Different terms have been proposed to designate hepatocellular adenomas with features that are suggestive but not diagnostic for hepatocellular carcinoma, including atypical hepatocellular neoplasms, hepatocellular neoplasm of uncertain malignant potential, and well-differentiated hepatocellular neoplasm with atypical or borderline features [25, 26].

Our study includes cases sent by the originating pathologists for consultative second opinions and four cases previously reported as either single case reports or part of a case series $[6,8,27]$. Upon review of the key histologic sections, the primary authors considered all of these lesions represent borderline lesion or hepatocellular carcinoma arising in liver fatty acid binding protein-deficient hepatocellular adenomas primarily on the basis of architectural and cytologic atypia. The natural history of these lesions, however, is not aggressive and corresponds well to the low-grade histology. At the time of diagnosis, none of the patients presented with metastatic disease and only one patient $(8 \%)$ had disease recurrence during the follow-up period. Whereas, BioulacSage et al. [22] reported recurrence and death in 33\% and $50 \%$ of $\beta$-catenin mutated hepatocellular adenoma patients with malignant transformation $(n=6)$, respectively.

Predisposing factors of malignant transformation in liver fatty acid binding protein-deficient hepatocellular adenoma are not well-established because of the low number of cases. In contrast to $\beta$-catenin mutated hepatocellular adenoma patients, liver fatty acid binding protein-deficient hepatocellular adenoma patients with malignant transformation were predominantly female $(p=0.018)$ with multiple adenomas $(p=0.0009)$. Of these patients, a subset of them (39\%) showed a background of hepatic adenomatosis. Other underlying disorders seen in our patient cohort included Budd-Chiari syndrome, possible Osler-Weber-Rendu syndrome, maturity-onset diabetes of the young type 3 , and type 2 diabetes mellitus. Patient 8 was confirmed to have maturity-onset diabetes of the young type 3 due to germline mutations of HNFIA. The genetic testing was not performed in other patients at the time of writing. Various hepatic lesions including different subtypes of hepatocellular adenoma (HNF1A-inactivated hepatocellular adenoma, $\beta$-catenin mutated hepatocellular adenoma, and 
inflammatory hepatocellular adenoma) have been reported in patients with vascular liver disease [7, 27]. In addition, hepatocellular carcinoma has been associated with Budd-Chiari syndrome, particularly in patients with obstruction of the hepatic portion of the inferior vena cava [28]. Although the pathogenesis is unclear, it is believed that hepatic venous obstruction would lead to hepatic injury, fibrosis, and cirrhosis which contribute to carcinogenesis in patients with Budd-Chiari syndrome [29]. In addition to the previous case reported by Stueck et al. [6] (included in the current study), Willson et al. [9] reported malignant transformation of $H N F 1 A$-inactivated hepatocellular adenoma in a family member of maturity-onset diabetes of the young type 3. This familial form of diabetes is inherited in autosomal dominant fashion, secondary to germline mutations of $H N F 1 A$, and often associated with hepatic adenomatosis $[5,9]$. Patient 9 was reported to have type 2 diabetes mellitus. Recently, type 2 diabetes mellitus is considered as a "new" risk factor for malignant transformation of hepatocellular adenoma along with high alcohol intake and fibrosis in non-tumoral liver [2]. However, the possibility of this patient having maturity-onset diabetes of the young type 3 cannot be completely excluded, as this entity is often misdiagnosed as type 2 diabetes mellitus [30] and her daughter also had a history of developing hepatocellular adenomas (unknown subtype).

Histologically, the adenoma component of most lesions in our study demonstrated a proliferation of normal-toslightly thickened hepatocyte plates with unpaired arterioles and decrease of steatosis in all cases. Distinguishing the hepatocellular adenoma from the hepatocellular carcinoma component can be challenging in such cases, and the diagnosis should not be based on the absence or presence of steatosis. In general, when hepatocellular carcinoma diagnosis has been established, the differentiation between the adenoma and carcinoma portions becomes arbitrary.

The morphologic criteria of borderline lesion/hepatocellular carcinoma used in the study are well-established, which include pseudoglandular architecture, cytologic, and architectural atypia [10, 31]. Pseudoglands are reportedly seen in high grade dysplastic nodules, $\beta$-catenin mutated hepatocellular adenoma (occasionally), and hepatocellular carcinoma [10, 32]. Therefore, focal pseudoglandular formation in the absence of cytologic and architectural atypia should not be interpreted as malignant transformation, particularly in $\beta$-catenin mutated hepatocellular adenoma. Cytologic atypia noted in our cases is characterized by small cell change with no significant nuclear pleomorphism. In the setting of prior bleeding, nuclear atypia should be interpreted with caution [32]. In our study, architectural atypia is the most consistent histologic finding to support the diagnosis of borderline lesion/carcinoma arising in hepatocellular adenomas. The presence of nodule-within- nodule appearance and thickened hepatocyte plates on $\mathrm{H} \& \mathrm{E}$ stains are helpful in confirming the diagnosis.

The absence of or minimal steatosis observed in all borderline/carcinoma components $(100 \%)$ is an important histologic feature to alert the pathologist to the possibility of malignant transformation in liver fatty acid binding proteindeficient hepatocellular adenoma. Moreover, the diagnosis of this subtype of hepatocellular adenoma could easily be missed in a biopsy or resection specimen of hepatic lesion without significant steatosis. This underlines the importance of including liver fatty acid binding protein in the immunohistochemical work up of well-differentiated hepatocellular lesions despite lack of steatosis, even though it is known that lack liver fatty acid binding protein could exist in hepatocellular carcinoma [33].

Other histologic features in the hepatocellular carcinoma portions of these lesions include myxoid change $(39 \%)$, peliosis (46\%), and sinusoidal dilatation (46\%). These changes can be observed in both hepatocellular adenoma and carcinoma components. Salaria et al. [34] reported a series of primary hepatic tumors showing myxoid change including both hepatocellular adenomas and hepatocellular carcinomas. Interestingly, these lesions also demonstrated loss of liver fatty acid binding protein expression. Although myxoid change does not necessarily imply malignant transformation, it might be a histologic signature of liver fatty acid binding protein-deficient hepatic tumors. Fujimoto et al. [35] reported that peliotic change can be seen in up to $39 \%$ of hepatocellular carcinomas. However, this histologic finding does not have any clinical significance other than altering ultrasonographic findings of hepatocellular carcinoma. It is also important to recognize that sinusoidal dilatation/telangiectasia is often identified in inflammatory hepatocellular adenoma [2, 5].

In our opinion, $H \& E$ and reticulin stains are the most reliable diagnostic tools to distinguish a well-differentiated hepatocellular carcinoma from hepatocellular adenoma. Loss or fragmentation of reticulin framework in the less fatty areas of the tumors is exclusively seen in hepatocellular carcinoma and not in other types of hepatocellular lesions [36-38]. Diffuse CD34 immunohistochemical staining pattern is also helpful to support the diagnosis of hepatocellular carcinoma. However, a similar staining pattern can be seen in $\beta$-catenin mutated hepatocellular adenoma (exon 3S45 and exon 7/8) [39, 40]; thus, correlation with morphologic findings is important. Swanson et al. [41] reported that the combination of intact reticulin with either glypican-3 or glutamine synthetase negativity shows a sensitivity and specificity of $92 \%$ and $95 \%$, respectively, to distinguish hepatocellular adenoma from hepatocellular carcinoma using tissue microarray. These stains, however, are not always helpful when used individually. Moreover, glypican-3 is more sensitive in poorly-differentiated 
hepatocellular carcinomas [42]. Therefore, its utility in the work-up of well-differentiated hepatocellular lesions is limited. In our study, the immunoreactivity of glypican-3 is identified only in one of six $(17 \%)$ well-differentiated hepatocellular carcinomas.

The combined use of glutamine synthetase and heatshock protein 70 has also been studied to distinguish typical hepatocellular adenoma from atypical hepatocellular neoplasms and well-differentiated hepatocellular carcinoma [43]. Positive staining of one of the markers is noted more frequently in hepatocellular carcinoma and atypical cases compared to typical hepatocellular adenomas. Diffuse glutamine synthetase immunoexpression is a surrogate marker of beta-catenin activation in exon 3 (non S45) [2, 22], and more recently the staining pattern has been described in more details taking into account the level of beta-catenin pathway activation [44]. A comprehensive description of different glutamine synthetase staining pattern according to beta-catenin mutation types corresponding to different levels of beta-catenin activation is currently in progress [45, 46].

Molecular confirmation of HNF1A inactivation is only available in three cases, while five cases demonstrate absence of TERT promoter and CTNNB1 gene (exon 3) mutations. Although the pathogenesis remains to be elucidated, HNF1A mutations might play a role in the carcinogenesis via a different mechanism and pathway than CTNNB1 mutations [8, 33]. Hechtman et al. [8] reported HNF1A mutations in hepatocellular carcinoma are associated with negative viral hepatitis status, mutually exclusive with CTNNB1 hotspot mutations and more commonly noted in female patients without cirrhosis. Case 13 demonstrated GNAS exon 8 mutation in both hepatocellular adenoma and hepatocellular carcinoma components. HNF1A inactivation was unfortunately not confirmed molecularly in this case (diagnosis was rendered on the basis of loss liver fatty acid binding protein staining in both adenoma and carcinoma components). GNAS mutations have previously been described in inflammatory hepatocellular adenoma and hepatocellular carcinoma, with a proposed tumorigenesis via activation of inflammatory pathways by STAT3 activation [46]. The role of GNAS mutations in HNF1A-inactivated hepatocellular adenoma has not been described.

The limitations of our study include the referral bias and incomplete follow-up information. The retrospective nature and limited molecular testing also limit the overall interpretation. Future opportunities include studies with a longer follow-up time and comprehensive molecular testing.

In summary, we described the largest series of liver fatty acid binding protein-deficient hepatocellular adenoma with malignant transformation. Clinicians and pathologists should be aware of this rare phenomenon. Malignant transformation in this particular subtype arises predominantly in female patients with multiple hepatic lesions. All foci of malignant transformation demonstrate features of borderline lesion/ well-differentiated hepatocellular carcinoma which is often challenging to distinguish from the hepatocellular adenoma component. Architectural and cytological atypia on $\mathrm{H} \& \mathrm{E}$ and reticulin stains are the most reliable features in rendering the diagnosis. Lack of steatosis is also a helpful feature to raise the possibility of malignant transformation. Other histologic features in both hepatocellular adenoma and borderline/carcinoma portions include myxoid change, peliosis, and sinusoidal dilatation.

Acknowledgements We thank Prof. Jessica Zucman-Rossi (INSERM, Paris, France) who performed the molecular analysis on cases 2, 3 , and 4.

\section{Compliance with ethical standards}

Conflict of interest The authors declare that they have no conflict of interest.

Publisher's note Springer Nature remains neutral with regard to jurisdictional claims in published maps and institutional affiliations.

\section{References}

1. Zucman-Rossi J, Jeannot E, Nhieu JT, Scoazec JY, Guettier C, Rebouissou S, et al. Genotype-phenotype correlation in hepatocellular adenoma: new classification and relationship with HCC. Hepatology. 2006;43:515-24.

2. Nault JC, Couchy G, Balabaud C, Morcrette G, Caruso S, Blanc $\mathrm{JF}$, et al. Molecular classification of hepatocellular adenoma associates with risk factors, bleeding, and malignant transformation. Gastroenterology. 2017;152:880-94.

3. Stoot JHMB, Coelen RJS, De Jong MC, Dejong CH. Malignant transformation of hepatocellular adenomas into hepatocellular carcinomas: a systematic review including more than 1600 adenoma cases. HPB. 2010;12:509-22.

4. Bioulac-Sage P, Balabaud C, Zucman-Rossi J. Subtype classification of hepatocellular adenoma. Dig Surg. 2010;27:39-45.

5. Dhingra S, Fiel MI. Update on the new classification of hepatic adenomas: clinical, molecular, and pathologic characteristics. Arch Pathol Lab Med. 2014;138:1090-7.

6. Stueck AE, Qu Z, Huang MA, Camprecios G, Ferrell LD, Thung SN. Hepatocellular carcinoma arising in an HNF-1 $\alpha$-mutated adenoma in a 23-year-old woman with maturity-onset diabetes of the young: a case report. Semin Liver Dis. 2015;35:444-9.

7. Arrive L, Zucman-Rossi J, Balladur P, Wendum D. Hepatocellular adenoma with malignant transformation in a patient with neonatal portal vein thrombosis. Hepatology. 2016;64:675-7.

8. Hechtman JF, Abou-Alfa GK, Stadler ZK, Mandelker DL, Roehrl MHA, Zehir A, et al. Somatic HNF1a mutations in the malignant transformation of hepatocellular adenomas: a retrospective analysis of data from MSK-IMPACT and TCGA. Hum Pathol. 2019;83:1-6.

9. Willson JS, Godwin TD, Wiggins GA, Guilford PJ, McCall JL. Primary hepatocellular neoplasms in a MODY3 family with a novel HNF1A germline mutation. J Hepatol. 2013;54:904-7.

10. Park YN. Update on precursor and early lesions of hepatocellular carcinomas. Arch Pathol Lab Med. 2011;135:704-15.

11. Edmonson HA, Steiner PE. Primary carcinoma of the liver. Cancer. 1954;7:462-503. 
12. Kang HJ, Jeong HJ, Kim SW, Yu E, Lee YJ, Kim SY, et al. Hepatocellular carcinoma arising in a huge hepatocellular adenoma with bone marrow metaplasia. J Pathol Tansl Med. 2018;52:226-31.

13. Lunse S, Doring P, Heidecke CD, Partecke LI. Giant Hepatocellular carcinoma with bone metastasis in a young adult, emerged from pigmented adenoma with beta-Catenin activation: a case report. Int J Surg Case Rep. 2017;36:18-21.

14. Kwok WY, Hagiwara S, Nishida N, Watanabe T, Sakurai T, Ida $\mathrm{H}$, et al. Malignant transformation of hepatocellular adenoma. Oncology. 2017;92:16-28.

15. Sempoux C, Bisig B, Couchy G, Balabaud C, Zucman-Rossi J, Bioulac-Sage P. Malignant transformation of a $\beta$-catenin inflammatory adenoma due to an S45 $\beta$-catenin-activating mutation present 12 years before. Hum Pathol. 2017;62:122-5.

16. Mounajjed T, Yasir S, Aleff PA, Torbenson MS. Pigmented hepatocellular adenomas have a high risk of atypia and malignancy. Mod Pathol. 2015;28:1265-74.

17. Kim GJ, Seok JY, Rhee H, Choi JY, Choi JS, Kim KS, et al. $\beta$-catenin activated hepatocellular adenoma: a report of three cases in Korea. Gut Liver. 2014;8:452-8.

18. Calderaro J, Labrune P, Morcrette G, Rebouissou S, Franco D, Prevot S, et al. Molecular characterization of hepatocellular adenomas developed in patients with glycogen storage disease type 1 . J Hepatol. 2013;58:350-7.

19. Bellamy CO, Maxwell RS, Prost S, Azodo IA, Powell JJ, Manning JR. The value of immunophenotyping hepatocellular adenomas: consecutive resections at one UK centre. Histopathology. 2013;62:431-45.

20. Hechtman JF, Raoufi M, Fiel MI, Taouli B, Facciuto M, Schiano TD, et al. Hepatocellular carcinoma arising in a pigmented telangiectatic adenoma with nuclear $\beta$-catenin and glutamine synthetase positivity: case report and review of the literature. Am J Surg Pathol. 2011;35:927-32.

21. Farges O, Ferreira N, Dokmak S, Belghiti J, Bedossa P, Paradis V. Changing trends in malignant transformation of hepatocellular adenoma. Gut. 2011;60:85-9.

22. Bioulac-Sage P, Reboissou S, Thomas C, Blanc JF, Saric J, Sa Cunha A, et al. Hepatocellular adenoma subtype classification using molecular markers and immunohistochemistry. Hepatology. 2007;46:740-8.

23. Henriet E, Abou Hammoud A, Dupuy JW, Dartigues B, Ezzoukry Z, Dugot-Senant N, et al. Argininosuccinate synthase 1 (ASS1): a marker of unclassified hepatocellular adenoma and high bleeding risk. Hepatology. 2017;66:2016-28.

24. European Association for the Study of the Liver. EASL clinical practice guidelines on the management of benign liver tumours. $\mathbf{J}$ Hepatol. 2016;65:386-98.

25. Choi WT, Kakar S. Atypical hepatocellular neoplasms: review of clinical, morphologic, immunohistochemical, molecular, and cytogenetic features. Adv Anat Pathol. 2018;25:254-62.

26. Bedossa P, Burt AD, Brunt EM, Callea F, Clouston AD, Dienes $\mathrm{HP}$, et al. Well-differentiated hepatocellular neoplasm of uncertain malignant potential: proposal for a new diagnostic category. Hum Pathol. 2014;45:658-60.

27. Sempoux C, Paradis V, Komuta M, Wee A, Calderaro J, Balabaud $\mathrm{C}$, et al. Hepatocellular nodules expressing markers of hepatocellular adenomas in Budd-Chiari syndrome and other rare hepatic vascular disorders. J Hepatol. 2015;63:1173-80.

28. Park H, Yoon JY, Park KH, Kim DY, Ahn SH, Han KH, et al. Hepatocellular carcinoma in Budd-Chiari syndrome: a single center experience with long-term follow-up in South Korea. World J Gastroenterol. 2012;18:1946-52.

29. Liu FY, Wang MQ, Duan F, Fan QS, Song P, Wang Y. Hepatocellular carcinoma associated with Budd-Chiari syndrome: imaging features and transcatheter arterial embolization. BMC Gastroenterol. 2013;13:105.

30. Pihoker C, Gilliam LK, Ellard S, Dabelea D, Davis C, Dolan LM, et al. Prevalence, characteristics and clinical diagnosis of maturity onset diabetes of the young due to mutations in HNF1A, HNF4A, and glucokinase: results from the SEARCH for diabetes in youth. J Clin Endocrinol Metab. 2013;98:4055-62.

31. Kakar S, Grenert JP, Paradis V, Pote N, Jakate S, Ferrell LD. Hepatocellular carcinoma arising in adenoma: similar immunohistochemical and cytogenetic features in adenoma and hepatocellular carcinoma portions of the tumor. Mod Pathol. 2014;27:1499-509.

32. Sempoux C, Balabaud C, Bioulac-Sage P. Malignant transformation of hepatocellular adenoma. Hepat Oncol. 2014;1:421-31.

33. Cho SJ, Ferrell LD, Gill RM. Expression of liver fatty acid binding protein in hepatocellular carcinoma. Hum Pathol. 2016; 50:135-9.

34. Salaria SN, Graham RP, Aishima S, Mounajjed T, Yeh MM, Torbenson MS. Primary hepatic tumors with myxoid change: morphologically unique hepatic adenomas and hepatocellular carcinomas. Am J Surg Pathol. 2015;39:318-24.

35. Fujimoto M, Nakashima O, Komuta M, Miyaaki T, Kojiro M, Yano H. Clinicopathological study of hepatocellular carcinoma with peliotic change. Oncol Lett. 2010;1:17-21.

36. Hytiroglou P. Well-differentiated hepatocellular nodule: making a diagnosis on biopsy and resection specimens of patients with advanced stage chronic liver disease. Semin Diagn Pathol. 2017;34:138-45.

37. Sempoux C, Balabaud C, Paradis V, Bioulac-Sage P. Hepatocellular nodules in vascular liver diseases. Virchows Arch. 2018;473:33-44.

38. Singhi AD, Jain D, Kakar S, Wu TT, Yeh MM, Torbenson M. Reticulin loss in benign fatty liver: an important diagnostic pitfall when considering a diagnosis of hepatocellular carcinoma. Am J Surg Pathol. 2012;36:710-5.

39. Cappellen D, Balabaud C, Bioulac-Sage P. A difficult cas of $\beta$ catenin-mutated hepatocellular adenoma: a lesson for diagnosis. Histopathology. 2019;74:355-7.

40. Bioulac-Sage P, Sempoux C, Balabaud C. Hepatocellular adenoma: classification, variants and clinical relevance. Semin Diagn Pathol. 2017;34:112-25.

41. Swanson BJ, Yearsley MM, Marsh W, Frankel WL. A triple stain of reticulin, glypican-3, and glutamine synthetase: a useful aid in the diagnosis of liver lesions. Arch Pathol Lab Med. 2015;139:537-42.

42. Shafizadeh N, Ferrell LD, Kakar S. Utility and limitations of glypican-3 expression for the diagnosis of hepatocellular carcinoma at both ends of the differentiation spectrum. Mod Pathol. 2008;21:1011-8.

43. Nguyen TB, Roncalli M, Di Tommaso L, Kakar S. Combined use of heat-shock protein 70 and glutamine synthetase is useful in the distinction of typical hepatocellular adenoma from atypical hepatocellular neoplasms and well-differentiated hepatocellular carcinoma. Mod Pathol. 2016;29:283-92.

44. Rebouissou S, Franconi A, Calderaro J, Letouze E, Imbeaud S, Pilati C, et al. Genotype-phenotype correlation of CTNNB1 mutations reveals different beta-catenin activity associated with liver tumor progression. Hepatology. 2016;64:2047-61.

45. Balabaud C, Sempoux C, Gouw AS, Cappellen D, Bisig B, Bail $\mathrm{BL}$, et al. Patterns of glutamine synthetase expression as marker of beta catenin activation in hepatocellular adenomas. J Hepatol. 2018;68(Suppl.):S420-1.

46. Nault JC, Fabre M, Couchy G, Pilati C, Jeannot E, Tran Van Nhieu J, et al. GNAS-activating mutations define a rare subgroup of inflammatory liver tumors characterized by STAT3 activation. J Hepatol. 2012;56:184-91. 